\title{
Evaluation of Processing Parameters for Densification of Composite Propellants
}

Ana Carolina Marotti1,*, Maurício Ferrapontoff Lemos², Jonas Farias Santos²,3, Letivan Gonçalves de Mendonça-Filho ${ }^{1}$ Jakler Nichele ${ }^{1}$

Marotti AC DiD https://orcid.org/0000-0003-4217-671X

Lemos MF (D) https://orcid.org/0000-0002-5933-9135

Santos JF (D) https://orcid.org/0000-0001-8969-1300

Mendonça-Filho LG (iD https://orcid.org/0000-0002-3175-8477

Nichele J (D) https://orcid.org/0000-0003-3046-8459

\section{How to cite}

Marotti AC; Lemos MF; Santos JF; Mendonça-Filho LG; Nichele $J$ (2019) Evaluation of Processing Parameters for Densification of Composite Propellants. I Aerosp Technol Manag, 11, Special Edition: 11-14. https://doi. org/10.5028/jatm. etmq. 72

ABSTRACT: The present work investigated the effects of varying two parameters of a pilot-scale composite propellant plant: the mechanical vibration and the vacuum level. The application of mechanical vibration to the casted mold after the end of mixing has improved the propellant density. On the other hand, the change of the vacuum level had no significant effect. Static firing tests showed an increase in the gas generation rate with the increase of the number of voids.

KEYWORDS: Composite propellants; Densification; Vibration effects; Vacuum effects.

\section{INTRODUCTION}

Propellants are mixtures of substances that react in a deflagration regime when correctly initiated by external stimulus, generating gases at high temperatures and pressures, which can be used for the development of ballistic work of rockets and projectiles. Among the different types of propellants, the composites, are solid propellants whose oxidizer is generally an inorganic oxygenated and particulate salt, which is dispersed in a combustible plastic matrix, forming a uniform mass with rigid and rubbery aspect (Valença et al. 2013).

Among the characteristics required for solid propellants, high density is fundamental, since it directly affects the burning speed (Meyer et al. 2007), as well as the homogeneity of the final product, since the irregularity of the distribution of possible internal defects changes the instantaneous burning surfaces and consequently the flame propagation pattern (Valença et al. 2013).

The most common problems found from improper processing parameters are voids and microfractures inside the grain. Due to the cross-linking in the polymeric matrix, which is responsible for giving solid character to the composite propellant, the grain becomes susceptible to accidental breakage under operating conditions. In addition, the increase of internal defects facilitates the progressive propagation of failures. Furthermore, the irregularity of the macroscopic structure of the solid mass unsettles the burning of the grain and can lead to a substantial risk of explosion. Drying processes are applied in the preparation of raw materials, such as aluminum, and hydrophilic substances, such as liquid

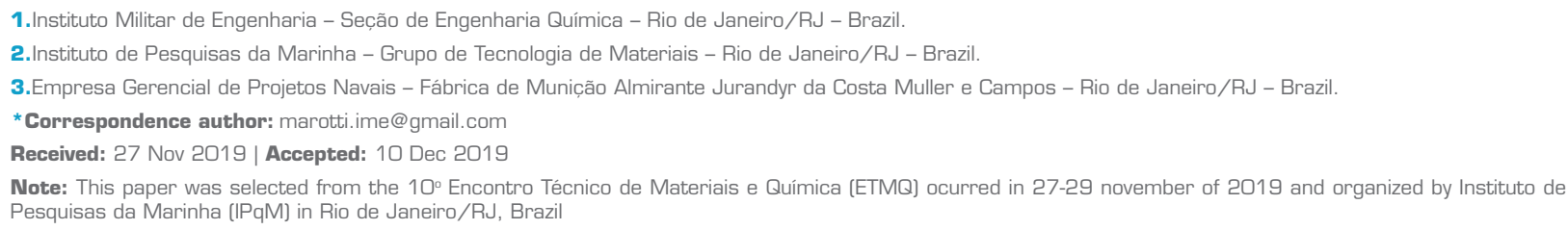


hydroxylated polybutadiene (HTPB) and dioctyl adipate (DOA) (Valença et al. 2013), to avoid their degradation and unwanted interactions with curing agents containing the organic function isocyanate, which can generate gaseous compounds that, in turn, produce voids in the grain by remaining trapped in the reticulate (Smith 1960).

The use of vacuum in the pouring stage favors the flow and accommodation of mass due to the pressure gradient while air and other gases are removed (MTCR 1996).

Thus, the main objective of this work is to investigate the effects on the final product caused by the variation of processing parameters. The definition and evaluation of parameters are focused on improving the manufacturing process of composite propellant by reducing voids and increasing grain thickening.

\section{METHODOLOGY}

In order to develop this work, a composite formulation containing the raw materials typically used was prepared: inorganic particulate solid oxidizer, aluminum powder and polymeric matrix composed of HTPB, plasticizer and polyamine bonding agent, and cured by diisocyanate. The material was homogenized in an orbital sigma type mixer for $6 \mathrm{~h}$ at $60^{\circ} \mathrm{C}$ under constant vacuum, first adding the liquid materials and then the solids, and evaluating the effect of two negative pressures in the process, generically called medium vacuum $(53.33 \mathrm{kPa})$ and high vacuum $(2.00 \mathrm{kPa})$, using the Maxpump and Edwards E-LAB 2 pumps respectively.

The mold was filled with the mixture under mechanical vibration during 5 min to evaluate the effect of vibration. The filled mold was kept in an oven at $60^{\circ} \mathrm{C}$ for 7 days until complete cure. Three batches of composite propellant were produced, whose nomenclature refers to the parameters under evaluation adopted in the respective processing.

The density of the composite propellants were determined after the curing. The density was defined as the mean of the ten measurements series obtained in Micromeritics AccuPyc 1330 helium pycnometer, under the following conditions: initial pressure of $19.50 \mathrm{psig}$ and equilibrium rate of $0.0050 \mathrm{psig} / \mathrm{min}$. Three $15 \mathrm{~mm}$ diameter cylindrical specimens were obtained from each of the three cured blocks.

Static firing tests were performed with the three grains of composite produced on the test bench shown in Fig. 1. The grain has been positioned on the split metal unit, the lower part of which is fixed to the bench, the upper part is removable and the back is perforated. Two devices containing mixed pyrotechnic drive were used, placed in the central drilling of the grain. The tests were conducted by remote triggering and recorded with high speed camera.

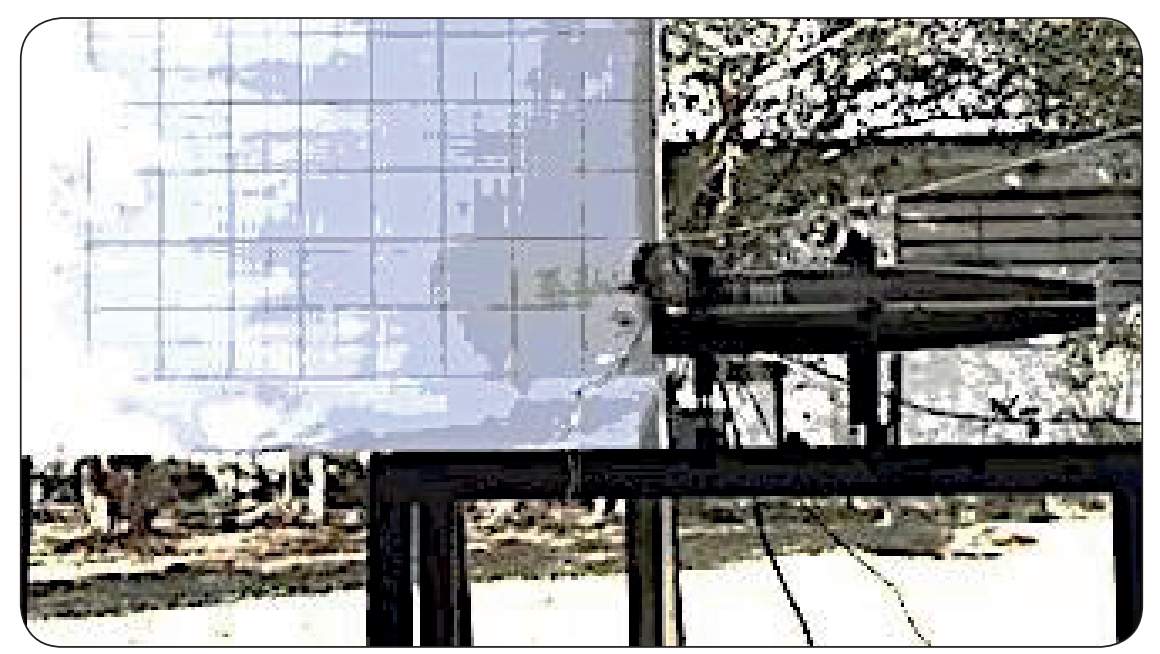

Figure 1. Static-firing test stand. 


\section{RESULTS AND DISCUSSION}

The results of the gas pycnometer tests for the produced propellants are presented in Table 1. The values obtained for the density showed that the application of mechanical vibration in the loaded mold produced the expected densification, consistent with the theory and practical experience already consolidated for the processing of other types of particle reinforced composites, such as concrete.

Table 1. Pycnometer density mass of composite propellants.

\begin{tabular}{|c|c|c|c|c|}
\hline Propellant & Densification & Vacuum level & Mean density $\left[\mathbf{g} / \mathbf{c m}^{\mathbf{3}} \mathbf{l}\right.$ & $\Delta .80$ \\
\hline highvac_vib & Yes & High & $1.5129 \pm 0.0092$ & 4.82 \\
\hline highvac_no_vib & No & High & $1.4982 \pm 0.0003$ & 4.13 \\
\hline medvac_vib & Yes & Mean & $1.5090 \pm 0.0081$ & \\
\hline
\end{tabular}

The densities of the propellants highvac_vib and highvac_no_vib are different due to the densification step. Considering that both grains are of the same volume, there is an increase of $0.0148 \mathrm{~g} / \mathrm{cm}^{3}$ of the density of the first in relation to the second, meaning an increase of $1 \%$ in the mass of material available to react during burning.

The densities experimentally measured were compared with the value estimated from the density of the components considered separately. The maximum theoretical density $\left(d_{\text {teo }}\right)$ resulted in $1.574 \mathrm{~g} / \mathrm{cm}^{3}$, which was computed using:

$$
d_{t e o}=\frac{1}{\sum \frac{x_{i}}{d_{i}}}
$$

where $d_{i}$ and $x_{i}$ are, respectively, the specific mass and mass fraction of each component of the composite formulation. This calculation maximizes the parameter, since it does not consider the fractions of different granulometries of ammonium perchlorate, whose packing results in formation of interstices, nor the intermolecular interactions between different components.

The differences between the values of actual and theoretical densities are related to the presence of voids caused by air bubbles and/or other gases produced in the reactions that occur in the processing steps. The percentage reduction of real specific masses in relation to the theoretically expected value $(\Delta d \%)$ was then evaluated using:

$$
\Delta d \%=\left(1-\frac{d_{p i c}}{d_{\text {teo }}}\right) \times 100 \%
$$

The ratio between the values of $\Delta d g$ was a result of the efficiency in attempting to remove remaining bubbles - from the mixing and loading steps - in the mass. Moreover, as the ammonium perchlorate is the component of greater volumetric fraction in the propellant, the factors responsible for hindering the mobility of particles of different granulometric fractions influence the accommodation of the mass inside the mold during pouring. The main factors are related to the friction forces between coarse particles, the mechanical encapsulation conferred by the cross-links in the polymeric matrix and the chemical bonds with bonding agent. In this way, the densification also contributed to achieve greater compactness between thick and thin particulates, reducing empty spaces.

The curves of the force exerted by the combustion gases (thrust) as a function of time for the composite propellant grains burned on a fixed bench are shown in Fig. 2. The absolute values of force cannot be published due to confidentiality issues. Thus, the thrust curves are presented as normalized values relative to the maximum force obtained in the tests.

The propellants highvac_vib, highvac_no_vib and medvac_vib displayed a decreasing burning profile, whose maximum point reached soon after the ignition, and experienced burning times of 29.6, 29.0 and 29.3 s respectively. The differences observed are consistent with the results of density presented in Table 1 and showed an increase in the gas generation rate with the number of 
voids or the decrease in specific mass as expected. The relation between the parameters is a consequence of the larger instantaneous burning surfaces due to internal macroscopic defects.

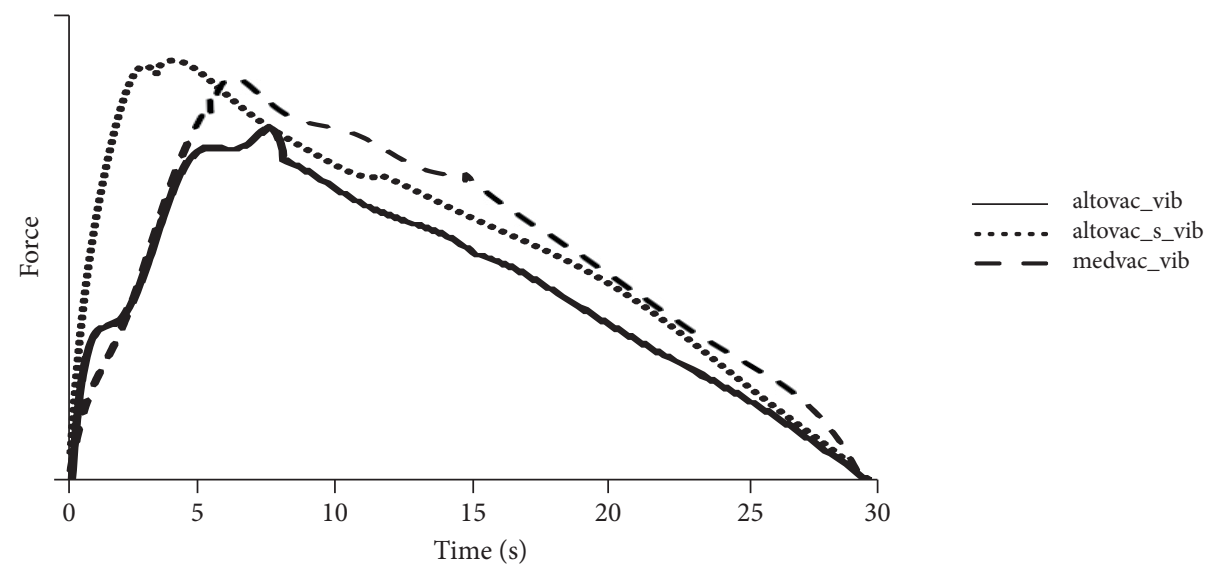

Figure 2. Force exerted by the combustion gases as a function of time.

\section{CONCLUSION}

The results obtained in the gas pycnometry tests and in the static-firing tests showed that the densification by mechanical vibration was effective and provided the reduction of the number of voids inside the finished propellant grains, while there was no significant difference between the effects of negative pressures of medium and high vacuum.

\section{FUNDING}

Empresa Gerencial de Projetos Navais (Emgepron).

\section{AUTHORS' CONTRIBUTION}

Conceptualization, Mendonça-Filho LG and Lemos MF; Methodology, Marotti AC, Mendonça-Filho LG and Nichele J; Research, Marotti AC, Santos JF and Lemos MF; Writing - First version, Marotti AC and Santos JF; Writing - Review \& Editing, Nichele J and Lemos MF; Funding Acquisition, Lemos MF; Supervision, Lemos MF, Mendonça-Filho LG and Nichele J.

\section{REFERENCES}

[MTCR] Missile Technology Control Regime (1996) MTCR Annex Handbook. Washington (United States): FAS.

Meyer R, Kohler J, Homburg A (2007) Explosives. 6th ed. Weinheim (Germany): Wiley-Vch.

Smith T (1960) Solid propellants...elastomeric-binder and mechanical-property requirements for solid propellants. Ind Eng Chem 52(9):776-780. https://doi.org/10.1021/ie50609a028

Valença US, Reis SS, Palazzo M, Rocha JF, Athayde AAC (2013) Engenharia dos explosivos: um enfoque dual. 1st ed. Rio de Janeiro (Brazil): Instituto Militar de Engenharia, Fundação Ricardo Franco. 